\title{
Mesh Size Effect in Numerical Simulation of Blast Wave Propagation and Interaction with Structures
}

\author{
SHI Yanchao(师燕超) ${ }^{1}$, LI Zhongxian(李忠献) ${ }^{1}$, HAO Hong(郝洪) ${ }^{2,1}$ \\ (1. School of Civil Engineering, Tianjin University, Tianjin 300072, China; \\ 2. School of Civil and Resource Engineering, the University of Western Australia, WA 6009, Australia)
}

\begin{abstract}
With the development of computer technology and computational mechanics, numerical method is becoming more and more popular in analysing the blast wave propagation and interaction with structures, since it is much cheaper and more convenient as compared with field tests. However, because of the extreme short duration of blast wave and energy transmission between different grids, it seems that the numerical results of blast wave propagation and interaction with structures are very sensitive to the finite element mesh size. Previous numerical simulation also shows that a mesh size acceptable to one blast scenario might not be proper for another case; even through the difference between the two scenarios is very small, indicating a simple numerical mesh size convergence test might not be enough to guarantee accurate numerical results. Therefore, a better understanding of the mesh size effect on numerical simulation of blast wave propagation and interaction with structures is critical. In this paper, both coarse mesh and fine mesh are used in different blast scenarios to investigate the mesh size effect on numerical results of blast wave propagation and interaction with structures. Based on the numerical results and their comparison with field test results and the design charts in TM5-1300, a numerical modification method is proposed to correct the influence of the mesh size effect on the simulated results. It can be easily used to improve the accuracy of the numerical results of blast wave propagation and blast loads on structures. Using this approach, a coarser mesh size can be used in simulations, which substantially reduces the computational time and computer memory.
\end{abstract}

Keywords: mesh size effect; numerical simulation; sensitivity; blast wave propagation; blast load; structures

\section{Introduction}

With the increasing interest in studying the blast induced damage and collapse of building structures worldwide, numerical simulation is becoming an important tool for predicting the blast wave propagation in urban environment, subway station, tunnels, as well as the blast load acting on structures ${ }^{[1-4]}$. When conducting numerical simulations, the accuracy of numerical results is strongly dependent on the mesh size used. Usually mesh size convergence test needs be carried out to find out the acceptable mesh size. The suitable mesh size for numerical analysis is blast scenario dependent. For example, a mesh size which is fine enough to predict the blast overpressure at a large scaled distance might become too coarse for the case when the scaled distance is small ${ }^{[5]}$. Therefore, unless mesh size convergence analysis is carried out for every blast scenario, it is impossible to determine the most efficient mesh size for a series of reliable numerical simulations. Moreover, because of the limitation of the computer and software, sometimes it is impossible to use a small mesh size in numerical simulation. In these cases, a large mesh size has to be used, although this will inevitably lead to some errors. Thus, a better understanding of the mesh size effect in numerical simulation of blast wave propagation and interaction with structures is important. To find an effective way to reduce this mesh size effect on the results of numerical simulations is also very important, which may lead to substantial savings in computational time and computer memory as using a relatively large mesh size becomes possible without compromise the accuracy of the numerical results.

Several researchers have studied the mesh size effect as part of their research of using numerical tool to study the blast wave propagation. Krauthammer and Otani ${ }^{[6]}$ studied the mesh, gravity and load effects on finite element simulations of blast loaded reinforced concrete structures. They made the conclusion that selecting a sufficiently fine mesh is very important for adequate definitions of deformations and stresses in blast loaded reinforced concrete structures. Luccioni, et al. ${ }^{[1]}$ discussed the mesh size effect when studying the blast loads assessment using hydro codes. They pointed out that a $10 \mathrm{~cm}$ mesh is accurate enough for the analysis of wave propagation in urban environments. A coarser mesh can be used to obtain qualitative results for comparison of the loads produced by different hypothetical blast events.

Accepted date: 2008-07-31

*Supported by National Natural Science Foundation of China (No.50638030 and No.50528808), the National Key Technologies R\&D Program of China (No.2006BAJ13B02), and the Australian Research Council (No.DP0774061).

SHI Yanchao, born in 1982, male, doctorate student.

Correspondence to LI Zhongxian, E-mail: zxli@tju.edu.cn. 
Even coarser meshes, up to $50 \mathrm{~cm}$, give a good estimation of the effects of moving the location of the explosive charges. Chapman, et $\mathrm{al}^{[5]}$ studied the mesh size effect of blast wave simulation using AUTODYN 2D. A grid size correction factor was proposed which allows for a correction of the simulated data if the grid is coarse. The correction factor could be used to eliminate the error a less than $10 \mathrm{~mm}$ coarse mesh might bring to the numerical results. Although these studies give some recommendations on selecting the proper mesh size, further study is still needed in this field because of the following reasons: 1) the mesh size effect on each blast load parameter is still unknown. Their relationships with scaled distance and standoff distance need be defined; 2) the correction factors proposed to eliminate the errors induced by coarse mesh are valid only when the mesh size is smaller than $10 \mathrm{~mm}^{[5]}$. In numerical simulation of a realistic blasting environment, often a mesh size larger than $10 \mathrm{~mm}$ has to be used. Therefore, correction methods for different mesh sizes are needed.

In this paper, the mesh size effect on each blast load parameter and its relationship with the scaled distance and standoff distance are studied through parametric simulations. Based on the numerical results, a new numerical modification method is proposed to eliminate the error in the numerical results induced by a coarse mesh.

\section{Mesh size effect}

This section is focused on the study of mesh size effect on blast load parameters, including the wave front arrival time, positive peak incident pressure, positive incident impulse, positive reflected pressure, and positive reflected impulse. Scaled distance and standoff distance are the parameters considered that might affect the mesh size effect.

\subsection{Blast incident pressure and impulse in free air burst explosion}

To study the mesh size effect on blast incident pressure and impulse, a free air burst explosion is simulated using AUTODYN. As the blast wave will move radically away from the centre of the explosion with a spherical wave front, utilization of symmetry conditions allows to represent the problem by a one-dimensional axisymmetrical model. This substantially reduces the computational effort. The one-dimensional (1D) "Wedge" mesh using axial symmetry in AUTODYN is utilized in this regard ${ }^{[7]}$.

Fig. 1 shows the configuration of the numerical model. Both the air and TNT explosive are modelled with Eulerian mesh. The detonation point is at the centre of the explosive. Gauge points are set up at different standoff distances to catch the incident pressure time histories at different scaled distances. The charge weight of TNT used is 1000 $\mathrm{kg}$. The scaled distance considered varies from 0.5 to $1.5 \mathrm{~m} / \mathrm{kg}^{1 / 3}$, which is the range that will cause severe outside this range to eliminate the wave reflection effect.damage to normal reinforced concrete structures. Outflow transmitting boundary condition is used outside this range to eliminate the wave reflection effect.
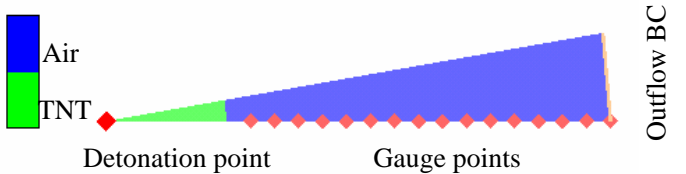

Fig. 1 Configuration of numerical model

Numerical simulations are carried out with different mesh sizes, namely, $5 \mathrm{~mm}, 10 \mathrm{~mm}, 25 \mathrm{~mm}$, $50 \mathrm{~mm}, 100 \mathrm{~mm}$ and $200 \mathrm{~mm}$. Fig. 2 shows the comparison of the incident pressure time histories at the scaled distance $1.0 \mathrm{~m} / \mathrm{kg}^{1 / 3}$. As shown, the rate of pressure rise from ambient to peak pressure becomes slower and the shape of the curve becomes flatter with the increase of the mesh size. The calculated peak pressure is also smaller when the mesh size is larger. The wave front arrival time, however, is independent of the mesh size, as expected.

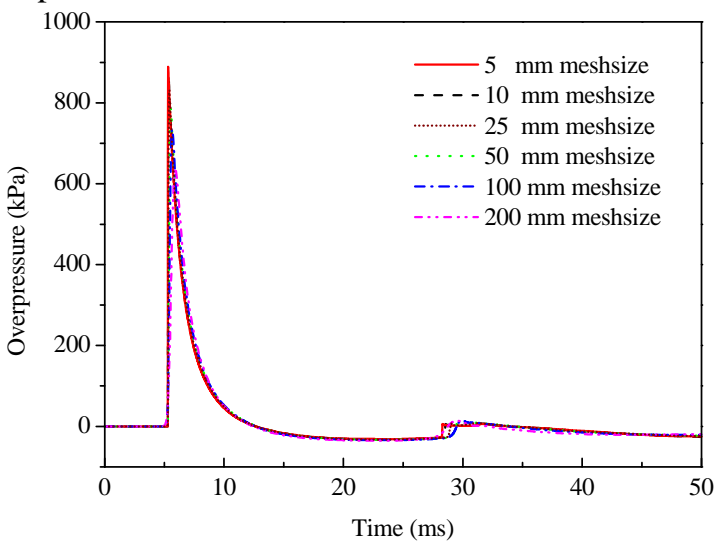

(a) Full range

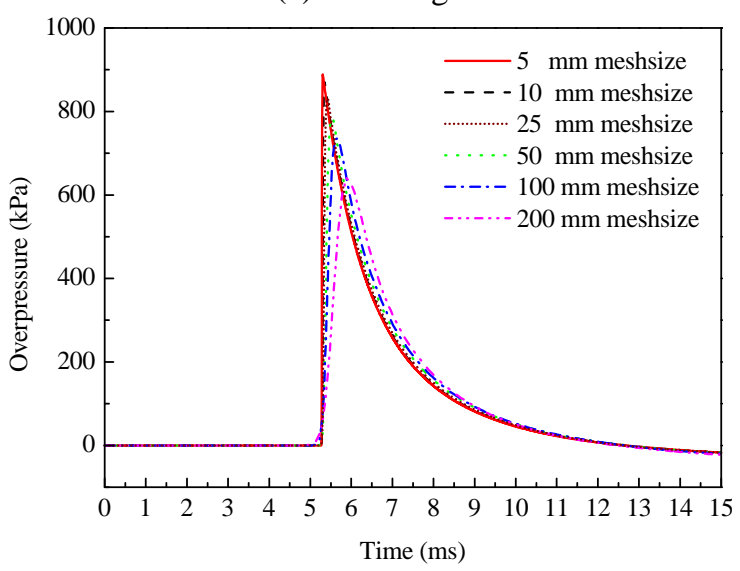

(b) Positive phase

Fig. 2 Comparison of pressure time histories obtained with different mesh sizes

The variation of the blast wave front arrival time, positive incident peak pressure and impulse with the scaled distance and mesh size are shown in Figs. 3-5, respectively. It is obvious in Fig.3 that the wave front arrival time is relatively independent of the mesh size. A $100 \mathrm{~mm}$ mesh size is good enough to provide accurate prediction of wave front 
arrival time of all the scaled distances considered in this study. As can be seen in Fig.4, for the positive incident peak pressure, when the scaled distance is less than $0.8 \mathrm{~m} / \mathrm{kg}^{1 / 3}$, even a small mesh size of 5 $\mathrm{mm}$ will fail to get the accurate estimation of the positive incident peak pressure as compared with that from TM5-1300 ${ }^{[8]}$. However, a coarser mesh size of $100 \mathrm{~mm}$ is The variation of the blast wave front arrival time, positive incident peak pressure and impulse with the scaled distance and mesh size are shown in Figs. 3-5, respectively. good enough to catch the positive incident peak pressure generated from blasts at a scaled distance of 2 $\mathrm{m} / \mathrm{kg}^{1 / 3}$. From Fig.5, one can see that the impulse is relatively independent of the mesh size, but the estimated positive impulse is smaller than that from TM5-1300. This observation is consistent with the numerical results derived by Chapman, et $\mathrm{al}^{[5]}$. This might be because of the difficulty of obtaining the accurate positive incident impulse in field tests on which TM5-1300 is based ${ }^{[8]}$. Thus, the convergent estimated positive impulse is assumed to be accurate.

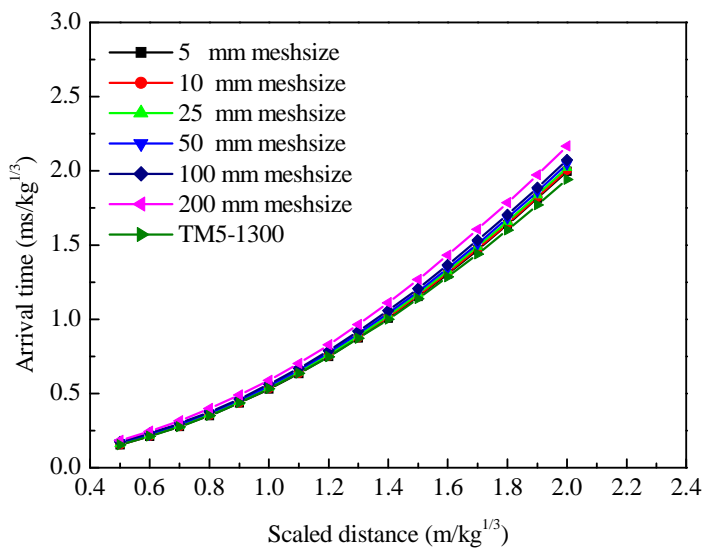

Fig. 3 Variation of the normalized wave front arrival time with mesh size and scaled distance

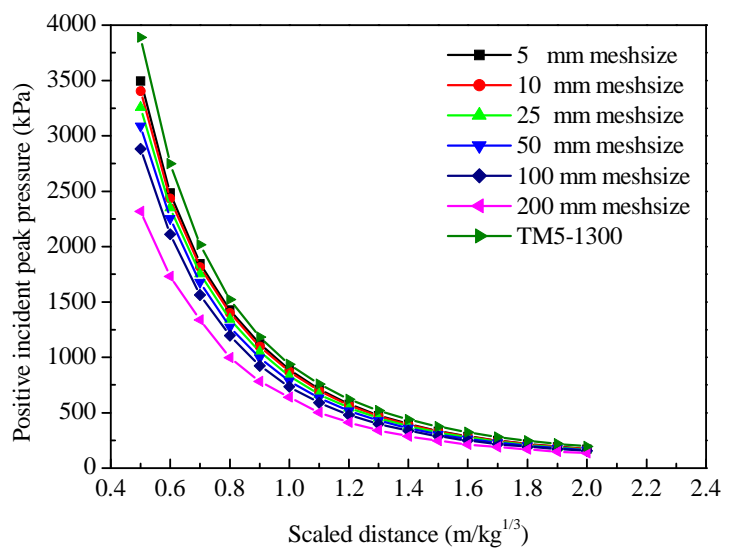

Fig. 4 Variation of the positive incident peak pressure with mesh size and scaled distance

In summary, the incident peak pressure is much more sensitive to the mesh size than the wave front arrival time and impulse. For both the incident peak pressure and impulse, the mesh size sensitivity decreases with the increase of the scaled distance.

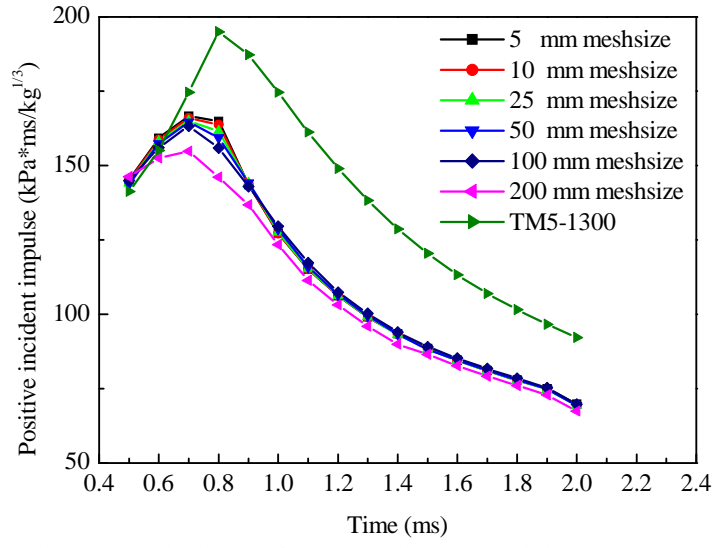

Fig. 5 Variation of normalized positive incident impulse with mesh size and scaled distance

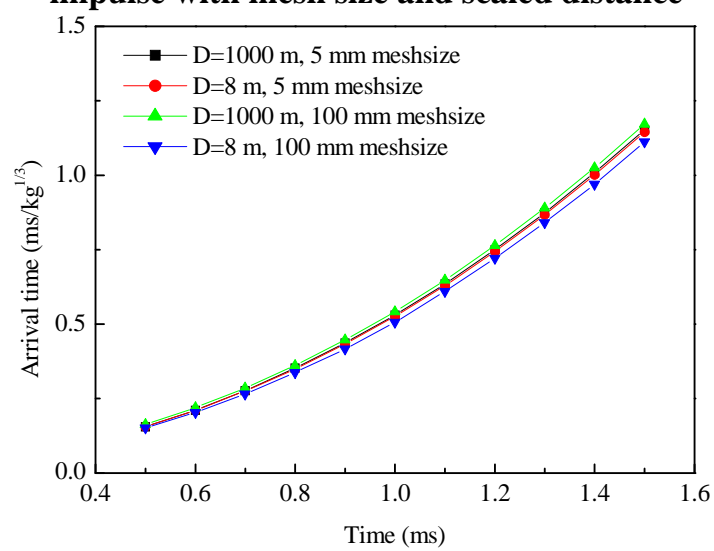

Fig. 6 Variation of the normalized wave front arrival time with mesh size and standoff distance

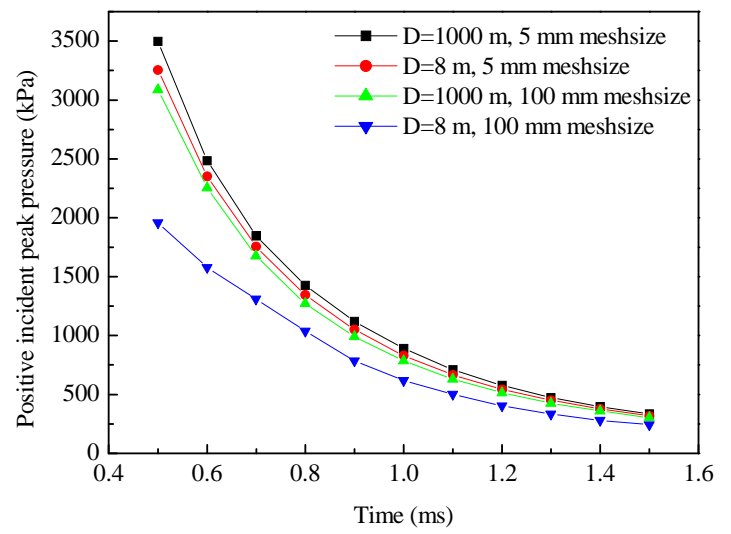

Fig. 7 Variation of the positive incident peak pressure with mesh size and standoff distance

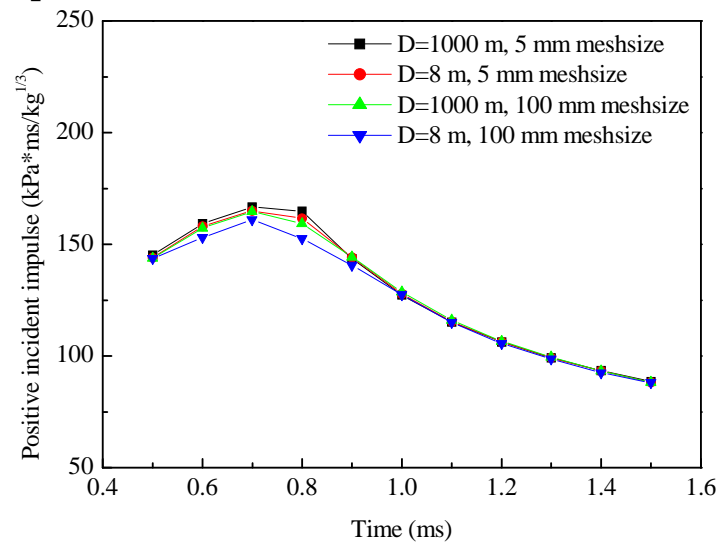

Fig. 8 Variation of normalized positive incident impulse with mesh size and standoff distance 
To study the mesh size effect on blast waves at different standoff distances, the same numerical model as shown in Fig.1 is established using AUTODYN, but only $8 \mathrm{~kg}$ TNT charge weight is considered. Therefore, to get the scaled distances between $0.5 \mathrm{~m} / \mathrm{kg}^{1 / 3}$ and $1.5 \mathrm{~m} / \mathrm{kg}^{1 / 3}$, the standoff distance only need to vary from $1 \mathrm{~m}$ to $3 \mathrm{~m}$, while in the previous simulation, the standoff distance should vary from $1000 \mathrm{~m}$ to $1500 \mathrm{~m}$ to get the scaled distances from $0.5 \mathrm{~m} / \mathrm{kg}^{1 / 3}$ to $1.5 \mathrm{~m} / \mathrm{kg}^{1 / 3}$. This numerical simulation is also carried out with different mesh size, i.e., $5 \mathrm{~mm}, 10 \mathrm{~mm}, 100 \mathrm{~mm}$ and $200 \mathrm{~mm}$. Comparisons between the results at the same scaled distance but different standoff distance from the above two simulations are made. Some typical ones are given in Figs. 6-8. Fig.6 shows the variation of the normalized wave front arrival time with mesh size and standoff distance while the scaled distance are all equal to $1.0 \mathrm{~kg} / \mathrm{m}^{1 / 3}$. As can be seen in the figure, when the standoff distance decreases from $1000 \mathrm{~m}$ to $8 \mathrm{~m}$, a $100 \mathrm{~mm}$ mesh size is not small enough for reliable numerical predication of wave front arrival time. At standoff distance $8 \mathrm{~m}$, the $100 \mathrm{~mm}$ mesh size results in smaller arrival time as compared to that from $5 \mathrm{~mm}$ mesh size. Fig.7 shows the predicted positive incident peak pressure. As shown, when the mesh size is the same, the predicted positive incident peak pressure at scaled distance $1.0 \mathrm{~kg} / \mathrm{m}^{1 / 3}$ decrease with the decrease of standoff distance. When the standoff distance is the same, the difference between the predicted positive incident peak pressures at same scaled distance from difference mesh size is enlarged with the decrease of standoff distance. This indicates that the predicted positive incident peak pressure is becoming more sensitive to mesh size with the decrease of the standoff distance. Fig. 8 shows the positive incident impulse. It is less sensitive to the standoff distance, as compared to the positive incident peak pressure.

\subsection{Blast reflected pressure from surface blast explosion}

The mesh size sensitivity for the positive reflected pressure and impulse is also investigated. As shown in Fig.9, 3D simulations are carried out to get the reflected pressure time histories on a structural component in a surface blast explosion. In the model, air and high explosive (TNT) are modelled with Eulerian mesh, while both rigid ground and rigid structural component are modelled using unused grids ${ }^{[7]} .8 \mathrm{~kg}$ equivalent charge weight of TNT is used. Standoff distance of $2 \mathrm{~m}$ and $3 \mathrm{~m}$ are selected to get the scaled distance of $1.0 \mathrm{~m} / \mathrm{kg}^{1 / 3}$ and $1.5 \mathrm{~m} / \mathrm{kg}^{1 / 3}$. The dimension of the structural component is considered to be $1200 \mathrm{~mm}$ wide, 400 $\mathrm{mm}$ deep and $1500 \mathrm{~mm}$ high. Since the symmetry of the whole blast scenario, only a quarter of which is modelled. Besides the two symmetry planes and one rigid ground, the boundary conditions of the other three planes are set to be outflow.
To reduce the computational time, a two-stage method is adopted. The first stage is to simulate the initial detonation in the first two meters by 2D simulation. The mesh size in this stage is $2 \mathrm{~mm}$. In the second stage, the result from the 2D simulation is remapped into a $3 \mathrm{D}$ model to generate a 3D blast wave, which will impinge on the structural component. In the $3 \mathrm{D}$ simulation, four different mesh sizes are utilized, they are $25 \mathrm{~mm}, 50 \mathrm{~mm}$, $100 \mathrm{~mm}$ and $200 \mathrm{~mm}$. Fig.10 gives the typical contours of blast pressure during the interaction between the blast wave and the structure component, in which the amplification of the reflected pressure is obvious.

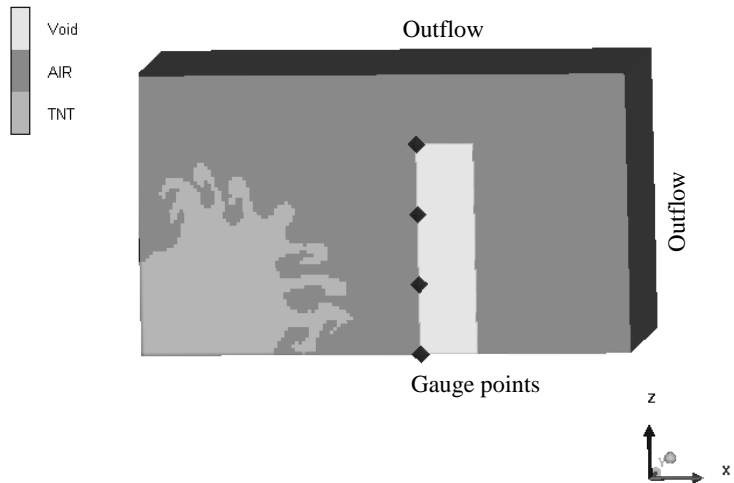

Fig. 9 Configuration of the $3 D$ numerical model

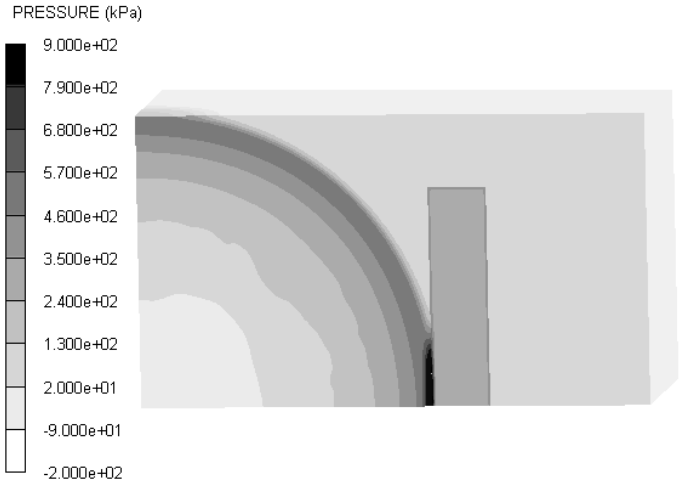

Fig. 10 Typical contours of reflected pressure

Only the reflected pressure time history at the base of the structure is analysed herein. The variation of the wave front arrival time, positive reflected peak pressure and positive reflected impulse and their comparisons with those obtained from TM5-1300 with different mesh sizes are given in table 1 and table 3 . Since the width of the structural component is $1.2 \mathrm{~m}$, the dimension effect of the structure on blast wave-structure interaction can be approximately ignored. Therefore, the results could be directly compared with those from TM5-1300. From these comparisons, one can find:

(1) The calculated positive reflected peak pressure and impulse are less sensitive to the mesh size with the increase of the scaled distance;

(2) Compared with the wave front arrival time and the positive reflected impulse, the positive reflected peak pressure is much more sensitive to the mesh size;

(3) By comparing data in Table 2 and Table 3, it is observed that the positive reflected peak 
pressure is more sensitive to the mesh size than the

positive incident peak pressure.

Table 1 variation of the wave front arrival time and positive reflected impulse with mesh size and their comparisons with those obtained from TM5-1300 ${ }^{[8]}$

\begin{tabular}{|c|c|c|c|c|c|c|c|c|}
\hline \multirow{2}{*}{$\begin{array}{l}\text { Mesh } \\
\text { size } \\
\text { mm }\end{array}$} & \multicolumn{4}{|c|}{ Scaled distance $=1.0 \mathrm{~m} / \mathrm{kg}^{1 / 3}$} & \multicolumn{4}{|c|}{ Scaled distance $=1.5 \mathrm{~m} / \mathrm{kg}^{1 / 3}$} \\
\hline & $\begin{array}{l}\text { Estimated } \\
\text { impulse } \\
(\mathrm{kPa} \bullet \mathrm{ms})\end{array}$ & error & $\begin{array}{l}\text { Estimated } \\
\text { arrival } \\
\text { time(ms) }\end{array}$ & $\begin{array}{l}\text { TM5- } \\
1300 \\
\text { (ms) }\end{array}$ & $\begin{array}{l}\text { Estimated } \\
\text { Impulse } \\
(\mathrm{kPa} \cdot \mathrm{ms})\end{array}$ & error & $\begin{array}{l}\text { Estimated } \\
\text { arrival } \\
\text { time(ms) }\end{array}$ & $\begin{array}{l}\text { TM5- } \\
1300 \\
\text { (ms) }\end{array}$ \\
\hline 5 & 1471 & $-16 \%$ & 0.85 & 0.92 & 989 & $-5 \%$ & 1.83 & 1.96 \\
\hline 10 & 1362 & $-23 \%$ & 0.82 & 0.92 & 937 & $-10 \%$ & 1.81 & 1.96 \\
\hline 25 & 1199 & $-32 \%$ & 0.82 & 0.92 & 846 & $-18 \%$ & 1.80 & 1.96 \\
\hline 50 & 948 & $-46 \%$ & 0.80 & 0.92 & 701 & $-33 \%$ & 1.80 & 1.96 \\
\hline
\end{tabular}

\section{Numerical modification: a method to eliminate coarse mesh error}

As discussed in the previous section, the wave front arrival time and positive incident/reflected impulse are less sensitive to the mesh size than the positive incident/reflected peak pressure, indicating acceptable results of wave front arrival time and positive incident /reflected impulse can be obtained with a coarse mesh size. However, a finer mesh size is needed to reach the same level of accuracy of the peak incident and reflected pressure. Another observation from the previous section is that when the mesh size is coarser, there will be a longer rising time in both the incident and reflected pressure time histories, which is not realistic as the pressure in fact rises almost instantly to the peak value in the true situations. .Thus, a numerical modification method to use the wave front arrival time, the positive incident/reflected impulse and the rise time to reconstruct the positive incident/reflected peak pressure is developed. In this case, the more accurate positive incident/reflected peak pressure are obtained through this modification method since a coarse mesh gives more accurate predictions of the positive incident/reflected impulse and wave front arrival time. Even if the mesh size is so coarser that it fails to reliably predict the positive incident/reflected impulse and wave front arrival time, the accuracy of the positive incident/reflected peak pressure can be improved through this method because of different mesh size sensitivity of these blast parameters.

Usually a fictitious triangular pressure pulses is used to approximate the positive pressure time history, as shown in Fig.11. With this simplification, the positive pressure time history is modelled by an abrupt rise of pressure to its peak value and a linear decay to the ambient pressure. The pulse can be defined by the arrival time $T_{\mathrm{a}}$, the fictitious duration of the positive phase $T_{0 \mathrm{f}}$ and the positive peak pressure $P$, as shown in Fig. 12.

According to the observation and above discussion, a numerical modification method is put forward. Some assumptions are made as follows:
(1) The mesh size is fine enough to catch the wave front arrival time and positive incident/reflected impulse;

(2) The nonzero rise time of the positive incident/reflected pressure time history is caused by the coarser mesh and should be deleted;

(3) The duration that the positive incident/reflected pressure decays from peak to zero is not sensitive to the mesh size.

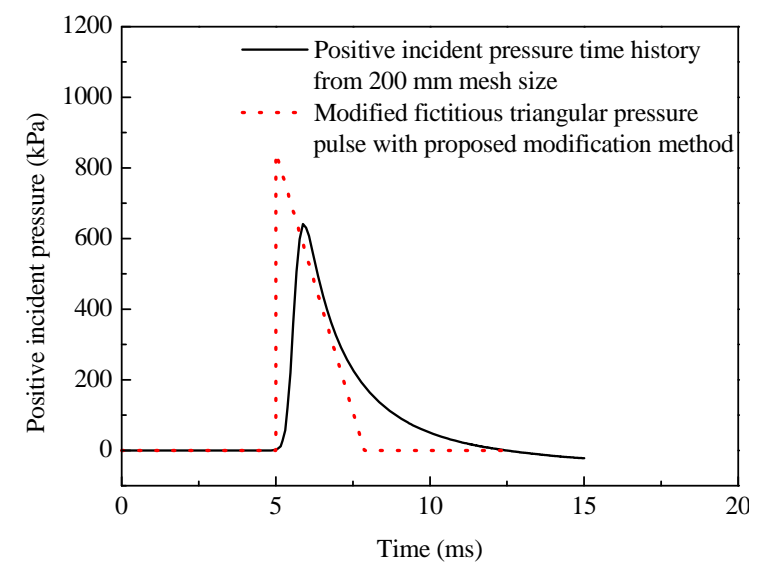

Fig. 11 Modified fictitious triangular pressure pulse for positive incident pressure time history from simulation with a $200 \mathrm{~mm}$ mesh size $(Z=1.0$

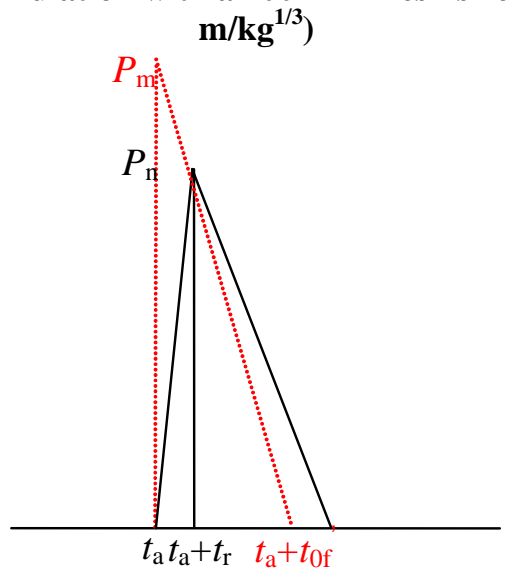

Fig. 12 Sketch of the numerical modification method

The procedure of the numerical modification method is:

(1) Derive the wave front arrival time $T_{\mathrm{a}}$, rise time $T_{\mathrm{r}}$, positive peak incident/reflected pressure $P_{\mathrm{n}}$ and impulse $I_{\mathrm{n}}$ from numerical simulation; 
(2) Simplify the pressure time history as a triangular pressure pulse, as shown in Fig. 12. The duration of the positive incident/reflected pressure is

$$
T_{0 \mathrm{f}}=2 I_{\mathrm{n}} / P_{\mathrm{n}}
$$

(3) The modified peak incident/reflected pressure can be obtained as

$$
\begin{gathered}
P_{\mathrm{m}}=2 I_{\mathrm{n}} / T_{0 \mathrm{f}}{ }^{\prime} \\
T_{0 \mathrm{f}}{ }^{\prime}=T_{0 \mathrm{f}}-T_{\mathrm{r}}
\end{gathered}
$$

Using this procedure, fictitious triangular pressure pulse could be obtained as the dot line shown in Fig.12, in which the accuracy of the positive peak incident/ reflected pressure is improved.

In order to validate the proposed numerical modification method, the above procedures are used to process the results from the numerical simulations carried out in section 1 . Fig. 11 shows the comparison of the modified fictitious triangular pressure pulse and positive incident pressure time history from numerical simulation of free air explosion with a $200 \mathrm{~mm}$ mesh size. As can be seen, the modified fictitious triangular pressure pulse have a higher positive peak incident pressure, while both the wave front arrival time and positive incident impulse keep unchanged. Table 2 gives comparisons of the numerical and modified positive peak incident pressure for the 2D free air burst explosion simulation with different mesh sizes. From the table one can easily see that after the modification, the errors of the predicted positive peak incident pressure, as compared to TM5-1300, are reduced significantly. Even the mesh size is 200 $\mathrm{mm}$, the error can be reduced to be less than $10 \%$, which is acceptable for engineering use. Table 3 shows comparisons of the numerical and modified positive peak reflected pressure for the 3D surface blast explosion simulation with different mesh sizes. Again, the errors as compared with TM5-1300 prediction are reduced significantly after the modification.

\begin{tabular}{|c|c|c|c|c|}
\hline \multirow{2}{*}{$\begin{array}{l}\text { Mesh size } \\
\mathrm{mm}\end{array}$} & \multicolumn{4}{|c|}{ TNT charge weight $W=1000 \mathrm{~kg}$, Standoff distance $D=10 \mathrm{~m}$, Scaled distance $Z=1.0 \mathrm{~kg} / \mathrm{m}^{1 / 3}$} \\
\hline & $\begin{array}{l}\text { Estimated positive } \\
\text { peak incident } \\
\text { pressure }(\mathrm{kPa})\end{array}$ & $\begin{array}{l}\text { Error compared with } \\
\text { TM5-1300 }\end{array}$ & $\begin{array}{l}\text { Modified positive } \\
\text { peak incident } \\
\text { pressure }(\mathrm{kPa})\end{array}$ & $\begin{array}{l}\text { Error compared with } \\
\text { TM5-1300 }\end{array}$ \\
\hline 5 & 889 & $-5.0 \%$ & 900 & $-3.8 \%$ \\
\hline 10 & 872 & $-6.8 \%$ & 893 & $-4.6 \%$ \\
\hline 25 & 831 & $-11.2 \%$ & 877 & $-6.3 \%$ \\
\hline 50 & 786 & $-16.0 \%$ & 867 & $-7.4 \%$ \\
\hline 100 & 735 & $-21.3 \%$ & 863 & $-7.8 \%$ \\
\hline 200 & 640 & $-31.6 \%$ & 844 & $-9.8 \%$ \\
\hline
\end{tabular}

Table 2 Comparison of the numerical and modified positive peak incident pressure for the 2D free air burst explosion simulation with different mesh sizes

\begin{tabular}{|c|c|c|c|c|c|c|c|c|}
\hline \multirow{2}{*}{$\begin{array}{l}\text { Mesh size } \\
\mathrm{mm}\end{array}$} & \multicolumn{4}{|c|}{ Scaled distance $=1.0 \mathrm{~kg} / \mathrm{m}^{1 / 3}$} & \multicolumn{4}{|c|}{ Scaled distance $=1.5 \mathrm{~kg} / \mathrm{m}^{1 / 3}$} \\
\hline & $\begin{array}{l}\text { Estimated } \\
\text { positive peak } \\
\text { reflected } \\
\text { pressure }(\mathrm{kPa})\end{array}$ & $\begin{array}{l}\text { error } \\
\text { to } \\
\text { TM5- } \\
1300 \\
\end{array}$ & $\begin{array}{l}\text { Modified } \\
\text { positive peak } \\
\text { reflected } \\
\text { pressure }(\mathrm{kPa})\end{array}$ & $\begin{array}{l}\text { error } \\
\text { to } \\
\text { TM5- } \\
1300\end{array}$ & $\begin{array}{l}\text { Estimated } \\
\text { positive peak } \\
\text { reflected } \\
\text { pressure }(\mathrm{kPa})\end{array}$ & $\begin{array}{l}\text { error } \\
\text { to } \\
\text { TM5- } \\
1300 \\
\end{array}$ & $\begin{array}{l}\text { Modified } \\
\text { positive peak } \\
\text { reflected } \\
\text { pressure }(\mathrm{kPa})\end{array}$ & $\begin{array}{l}\text { error } \\
\text { to } \\
\text { TM5- } \\
1300 \\
\end{array}$ \\
\hline 25 & 4695 & $-42 \%$ & 5810 & $-29 \%$ & 1800 & $29 \%$ & 2147 & $-15 \%$ \\
\hline 50 & 3504 & $-57 \%$ & 4586 & $-43 \%$ & 1440 & $43 \%$ & 1779 & $-30 \%$ \\
\hline 100 & 2436 & $-70 \%$ & 3079 & $-62 \%$ & 1073 & $58 \%$ & 1344 & $-46 \%$ \\
\hline 200 & 1381 & $-83 \%$ & 1648 & $-80 \%$ & 735 & $71 \%$ & 901 & $-64 \%$ \\
\hline
\end{tabular}

Table 3 Comparison of the numerical and modified positive peak reflected pressure for the 3D surface blast explosion simulation with different mesh sizes

\section{Conclusions}

Mesh size sensitivity for each blast load parameters and the relationship between the scaled distance and standoff distance is studied through a series of numerical simulations and comparisons between the results and those from TM5-1300. It was found that the positive peak incident/reflected pressure and impulse are less sensitive to the mesh size with the increase of the scaled distance. Compared with the wave front arrival time and the positive incident/reflected impulse, the positive incident/reflected pressure is more sensitive to the mesh size, and the positive reflected peak pressure is more sensitive than the positive incident peak pressure.

A modification method is proposed to modify the numerically simulated peak pressures. It is based on the assumption that the numerically simulated impulse is accurate and the pressure rise to its peak value instantly with a zero rise time. The method was validated by comparing the modified positive incident/reflected peak pressure with numerical data and those from TM5-1300. The results show that the proposed modification method can be easily used to improve the accuracy of the numerical results of the blast parameters even when a coarser mesh size is used in the simulation. 


\section{Refererences}

[1] Luccioni B, Ambrosini D, Danesi R. Blast load assessment using hydrocodes [J]. Engineering Structures, 2006, 28(12):1736-1744.

[2] Remennikov AM, Evaluation of blast loads on buildings in urban environment [C]. In: Proceedings of Eighth International Conference on Structures Under Shock and Impact, SUSI VIII, Mar 29-31 2004. Crete, Greece, 2004, 73-82.

[3] Shi Y, Hao H, Li Z-X. Numerical simulation of blast wave interaction with structure columns [J]. Shock Waves, 2007, 17(1):113-133.

[4] Smith PD, Rose TA. Blast wave propagation in city streets - an overview [J]. Progress in Structural Engineering and Materials, 2006, 8(1):16-28.

[5] Chapman TC, Rose TA, Smith PD. Blast wave simulation using autodyn2d: A parametric study [J]. International Journal of Impact Engineering, 1995, 16(5-6):777787.

[6] Krauthammer T, Otani RK. Mesh, gravity and load effects on finite element simulations of blast loaded reinforced concrete structures [J]. Computers \& Structures, 1997, 63(6):1113-1120.

[7] AUTODYN. Theory manual [M]. Century Dynamics, 2006.

[8] Technical Manual (TM5-1300). To resist the effect of accidental explosions [M]. Department of the Army, Navy and the Air force, Washington, DC, 1990. 